\title{
BER-Based Codebook Construction for MIMO-OFDM Precoded Spatial Multiplexing Systems
}

\author{
Feng She, Wen Chen, Hanwen Luo, and Xiaoting Yang \\ Department of Electronic Engineering \\ Shanghai Jiaotong University, Shanghai, P. R. China 200240 \\ Email: \{shefeng630;wenchen\}@sjtu.edu.cn
}

\begin{abstract}
This paper deals with design and performance analysis of transmit precoder optimization for MIMO-OFDM systems with limited feedback of channel state information (CSI). We assume that the receiver has perfect channel knowledge while the transmitter has only partial channel knowledge from limited feedback. We present a BER-based optimal codebook construction algorithm for MIMO-OFDM systems under average power constraint using the Lloyd algorithm. The proposed optimal precoder has the structure of joint precoding and power allocation. Closed form solution has been derived for power allocation in the sense of minimum BER for the MIMO-OFDM systems. Simulation results show that the BER-based codebook construction algorithm with hybrid design of power allocation and multi-mode beamforming can achieve better performance than those of equal power allocattion based codebooks in literature.
\end{abstract}

\section{INTRODUCTION}

Multiple-input multiple-output (MIMO) system is well motivated for wireless communication through fading channels because it has the potential to improve transmission rate or diversity gain. The performance of a multi-antenna system depends on the degree of channel state information (CSI) available at the transmitter and the receiver. However, in practical wireless systems, the transmitter could not obtain perfect CSI because of various factors, e.g., estimation error, feedback delay, feedback error, etc. For multi-antenna systems, even partial channel knowledge can be valuable in enhancing system performance [5]. Thus, exploiting channel partial CSI at the transmitter in a MIMO wireless systems has attracted great attention recently [6], [7].

Transmit CSI can enhance MIMO system performance by using precoder. CSI at transmitter can be obtained by feedback from receiver in frequency division duplexing systems or by measuring the reverse channel in time division duplexing systems. Generally, it is assumed that perfect CSI is available at the receiver while the transmitter only get partial CSI or imperfect CSI. There are two ways to exploit partial CSI [6], [10]. One is to use the statistical characteristic of CSI [5], [10]; The other is to use limited feedback bits which indexing CSI. The latter way is to first quantize the channel at the receiver, and then send back the limited bits indexing the quantized CSI which is called codebook. One can design off-line a codebook containing transmission candidates [10], [11]. The previous works on limited feedback CSI based transmit precoding are based on various criteria such as the average signal to noise ratio (SNR) [12], [13], the outage probability [14], [16], and the symbol error rate [17], etc. Optimal transmit algorithms for maximal capacity based limited feedback CSI have been investigated in [24]. However, those are all based on equal power allocation. On the other hand, the transmitter structure in the previous works do not coincide with the optimal transmitter structure in [18]- [22].

By dividing frequency-selective channels into an equivalent set of frequency-flat subchannels, orthogonal frequency division multiplexing (OFDM) has emerged as an attractive modulation scheme to handle frequency selective fading resulting from delay spreading by expanding the symbol duration [8], [9]. It is also an effective technique to combat inter-symbol interference (ISI) caused by wireless multi-path fading channel [9]. Our focus in this paper is to take the critical work of determining which CSI and by which way to be sent to the transmitter for MIMO-OFDM systems.

In this paper, we consider power allocation across different sub-channels for MIMO-OFDM systems with limited feedback, where the minimal BER-based codebook construction algorithm is investigated. We also propose hybrid precoding and power adaptive allocation algorithm for MIMO-OFDM with the constructed optimal codebook by spatial multiplexing.

The paper is organized as follows. We outline MIMOOFDM model and the problem statement in section II. In section III, the transmitter structure and feedback model are described. The minimum BER-based coodbook construction algorithm is presented in section IV. Section V proposes the optimal precoder design algorithm for MIMO-OFDM system with limited feedback. The simulation result are presented in section VI followed by the conclusion in section VII.

\section{SYSTEM MODEL}

We consider $N_{t}$ transmit-antennas and $N_{r}$ receive antennas MIMO-OFDM wireless communication system with spatial multiplexing, where $N_{c}$ subcarriers is employed in an OFDM symbol. By MIMO-OFDM system, frequency-selective channel can be decoupled into $N_{c}$ parallel MIMO frequency-flat channels. 
Assume that there are $M$ symbols to be transmitted per subcarrier, which is then bounded by $\min \left(N_{t}, N_{r}\right)$. Collect the $N_{c} M$ symbols transmitted over $N_{c} N_{t}$ subcarriers in a $N_{c} M \times 1$ vector $x$. The precoder is applied to each subcarrier to allocate power across the $N_{c}$ subcarriers and $N_{t}$ antennas. Therefore the precoder $\boldsymbol{F}$ is an $N_{c} N_{t} \times N_{c} M$ block diagonal matrix with $N_{c}$ blocks of $N_{t} \times M$ matrices $\boldsymbol{F}_{n}, n=$ $1, \cdots, N_{c}$.

In this context, we consider a MIMO block-fading channel model where the channel state remains quasi-statistic within a fading block on each subcarrier, but behaves independently across a different fading block. The frequency responses of the $N_{c}$ MIMO channels can be described by a $N_{c} N_{r} \times N_{c} N_{t}$ block diagonal channel matrix $\boldsymbol{H}$ with $N_{c}$ blocks of $N_{r} \times N_{t}$ matrices $\boldsymbol{H}_{n}, n=1, \cdots, N_{c}$. If the channel keeps quasi-static, the $N_{c} M \times 1$ received symbol vector $\mathbf{r}$ is

$$
\boldsymbol{r}=\boldsymbol{G H F x}+\boldsymbol{G n},
$$

where the receiver $\boldsymbol{G}$ is an $N_{c} M \times N_{c} N_{r}$ block diagonal matrix with $N_{c}$ blocks of $M \times N_{r}$ matrices $\boldsymbol{G}_{n}, n=1, \cdots, N_{c}$, and $\boldsymbol{n}$ is an $N_{c} M \times 1$ noise vector, which has the Gaussian statistics.

In MIMO-OFDM system, each subcarrier can be considered as a MIMO channel. Suppose $\boldsymbol{x}^{T}=\left[\begin{array}{lll}\boldsymbol{x}_{1}^{T} & \cdots & \boldsymbol{x}_{N_{c}}^{T}\end{array}\right], \boldsymbol{r}^{T}=$ $\left[\begin{array}{lll}\boldsymbol{r}_{1}^{T} & \cdots & \boldsymbol{r}_{N_{c}}^{T}\end{array}\right]$ and $\boldsymbol{n}^{T}=\left[\begin{array}{lll}\boldsymbol{n}_{1}^{T} & \cdots & \boldsymbol{n}_{N_{c}}^{T}\end{array}\right]$, where $\boldsymbol{x}_{k}, \boldsymbol{r}_{k}$ and $\boldsymbol{n}_{k}$ for $k=1, \cdots, N_{c}$ are respectively the transmitted symbol vector, the received symbol vector and the noise vector over the $k$ th MIMO channel. From (1), the output-input relation for each subcarrier can be individually written out. For $n=1, \ldots, N_{c}$, denote

$$
\boldsymbol{y}_{p}=\boldsymbol{H}_{n} \boldsymbol{F}_{n} \boldsymbol{x}_{n}+\boldsymbol{n}_{n} .
$$

Then for $n=1, \cdots, N_{c}$, we have

$$
\boldsymbol{r}_{n}=\boldsymbol{G}_{n} \boldsymbol{y}_{n}=\boldsymbol{G}_{n} \boldsymbol{H}_{n} \boldsymbol{F}_{n} \boldsymbol{x}_{n}+\boldsymbol{G}_{n} \boldsymbol{n}_{n}
$$

\section{Optimal Transmitter Structure}

The optimal structure of $\boldsymbol{F}$ is $\boldsymbol{F}=\boldsymbol{W} \Theta \Phi$ [18]- [22], where $\boldsymbol{W}$ is an $N_{c} N_{t} \times N_{c} M$ matrix denoting the precoding matrix and $\Phi$ is an $N_{c} M \times N_{c} M$ diagonal matrix with the diagonal entries obtained by water-filling principle based power allocation [24], which means that the transmit power is allocated across the $N_{c}$ subcarriers and $N_{t}$ antennas. $\Theta$ is an $N_{c} M \times N_{c} M$ unitary matrix and each entry of the matrix has the same modulus $1 / \sqrt{K}$. Thus $\Theta$ could be a normalized DFT matrix or Hadamard matrix. When matrix $\Theta$ has been set to be the DFT or the Walsh-Hadamard matrices, it could provide the desired fading diversity with minimum complexity.

We will combine precoding and power allocation to get optimal transmit structure see Fig. 1, which can be expressed as

$$
y_{n}=\boldsymbol{H}_{n} \boldsymbol{w}_{n} \Theta_{n} \sqrt{\Phi_{n}} x_{n}+n_{n} \quad 1 \leq n \leq N_{c}
$$

where $\boldsymbol{w}_{n}$ is an $N_{t} \times M$ matrix which denotes precoding matrix and $\Phi_{n}$ is an $M \times M$ diagonal matrix with the diagonal entries denoting the allocated power along each beamforming modes, and $\Theta_{n}$ is a normalized DFT matrix or Hadamard matrix.

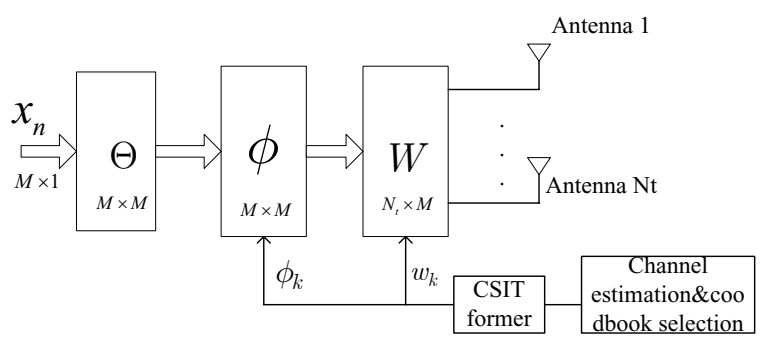

Fig. 1. Optimal transmitter structure

\section{Minimum BER-BAsed Codebook Construction}

In this section we manage to directly use the exact BER as the codebook design criterion. We first briefly outline the transmitter optimization in BER sense and then we will consider the codebook construction scheme that based on BER criterion. We assume that all subchannels carry the same number of bits and use the same constellation.

\section{A. Transmitter Optimization}

We now give the description of the transmitter optimization. It is important to remark that both the actual $Q$-function and Chernoff-bound are convex functions for a BER less than $2 \times$ $10^{-2}$. Thus, we can deal with actual Q-function and Chernoffbound as convex functions [20] for low BER.

Given an independent identical distribution block-fading MIMO channel with the transit CSI, our goal is to find the optimal performance based transmission scheme. Let $P_{e}$ denote the average BER of a MIMO-OFDM system, the optimal performance problem can be formulated as

$$
\left\{\begin{array}{l}
\min \quad P_{e} \\
\text { s.t. } E\left(\sum_{m=1}^{M} \operatorname{tr}\left(X_{m} X_{m}^{H}\right)\right) \leq P_{0}
\end{array}\right.
$$

where $X_{m}=\omega_{m} \sqrt{\phi_{m}} x_{m}$ and $P_{0}$ is the average transmit power.

\section{B. BER-Based Codebook Construction Algorithm}

The codebook construction for limited-rate feedback beamforming and precoding can be linked to a quantization problem [11]. We use Lloyd algorithm to search for good codewords based on BER criteria. The generalized Lloyd algorithm based codebook construction also provides an alterative systematic approach for the Grassmanian subspace packing problem [26].

In Lloyd algorithm, the quantizer quantizes the input space $\mathbb{C}^{N}$ into $N_{B}$ regions $\left\{R_{1}, \cdots, R_{N_{B}}\right\}$, where $R_{k}$ denote the cluster region of codeword $\omega_{k}$ for $k=1, \cdots, N_{B}$. Designing a quantizer means to find a codebook and a partition rule that jointly minimize the overall average distortion measure. The two necessary conditions are so-called centroid condition and nearest neighbor rule condition [27]. The centroid condition is to choose the optimal codeword by minimizing the average distortion over that region. The nearest neighbor rule is to 
determine the region where the vectors in this region are closer to the codeword of this region.

To design the BER criterion based codebook, we have to construct the nearest neighbor rule and the cenroid condition in this scenario. Thus, joint optimization of the channel region and the transmission modes is required. The two key works are

1) Given the channel vector region, find an optimal design of the transmission modes.

2) Given a set of transmission modes, find an optimal design of channel vector region.

Let $A_{k}$ denote the probability that the channel matrix $H$ lies in the region $R_{k} . \phi_{0}$ stands for the average transmit power. By jointly designing $\left\{R_{k}\right\}_{k=1}^{N_{B}}$ and transmitter strategy $\left\{\omega_{k}, \phi_{k}\right\}_{k=1}^{N_{B}}$, our ultimate goal is to

$$
\left\{\begin{array}{l}
\min J=\sum_{k=1}^{N_{B}}\left(A_{k} \cdot p_{e}(k)\right), \\
\text { s.t. } \sum_{k=1}^{N_{B}}\left(A_{k} \sum_{m=1}^{M}\left(\phi_{m}(k)\right)\right) \leq P_{0}
\end{array}\right.
$$

where $p_{e} \approx \frac{1}{M} \sum_{m=1}^{M}\left(0.2 e^{-g \gamma_{m}}\right)$ [23] denoting the average BER of QPSK modulation, and $\gamma_{m}$ is the system SNR of the $m$ th data stream. With the Lagrange multiplier $\mu$ for the average transmit power constraint, the distortion measure for optimizing the BER performance is given by

$$
\begin{aligned}
D\left(H, \omega_{k}, \phi_{k}\right)=\sum_{k=1}^{N_{B}} & \frac{A_{k}}{M} \sum_{m=1}^{M} 0.2 e^{-g \gamma_{m}(k)} \\
& +\mu\left(\sum_{k=1}^{N_{B}} A_{k} \sum_{m=1}^{M} \phi_{m}(k)-P_{0}\right)
\end{aligned}
$$

The distortion measure is a function of $\boldsymbol{H}, \omega_{k}$ and $\phi_{k}$, The partition index $k$ is sent to the transmitter, and the transmitter selects the optimal schemes in the codebook by the index $k$. Hence, the optimization problem could be solved by general Lloyd's algorithm, which can be outlined as following two steps.

S1 Given a certain channel condition regions of $\left\{R_{1}, \cdots, R_{N_{B}}\right\}$, find the optimal transmission scheme $\left\{\rho_{1}, \cdots, \rho_{N_{B}}\right\}$, where the transmitter scheme $\rho_{k} \triangleq\left\{\omega_{k}, \phi_{k}\right\}$ for $k=1, \cdots, N_{B}$ denoting the joint precoding matrice and power allocation along different modes. The optimal transmission scheme $\rho_{k}$, is given by the generalized region centroid condition. The optimal transmission strategy $\rho$, is given by the generalized region centroid condition.

$$
\rho_{k}^{o p t}=\arg \min _{\rho_{k}} E_{\left\{H \in R_{k}\right\}}\left(D\left(H, \rho_{k}\right)\right)
$$

S2 Given a transmission scheme $\left\{\rho_{k}\right\}$, find the optimal channel regions $R_{k}, 1 \leq k \leq N_{B}$. The optimal region is given by the nearest neighbor rule.

$$
R_{k}=\left\{H: D\left(H, \rho_{k}\right) \leq D\left(H, \rho_{j} ;\right), \forall j=1, \cdots, N_{B}\right\}
$$

By iteratively using centroid condition and the nearest neighbor rule, the overall distortion will decreases monotonically. We will use this principle to form a new codebook construction method. Here, we give the closed form solution of the two steps.

1) Solution of Step 1: For a given region $R_{k}, 1 \leq k \leq$ $N_{B}$, find the optimal $\rho_{k}$. For a region $R_{k}$, the probability $A_{k}$ and the channel covariance matrix can be calculated for each region. Assume that the eigen decomposition of $\boldsymbol{H}^{H} \boldsymbol{H}$ in region $R_{k}$ is $\boldsymbol{H}_{k}^{\boldsymbol{H}} \boldsymbol{H}_{k}=\boldsymbol{V}_{k} \Lambda_{k} \boldsymbol{V}_{k}^{H}$, where $\Lambda_{k}=$ $\operatorname{diag}\left(\lambda_{k, 1}, \cdots, \lambda_{k, N_{B}}\right)$ is an $N_{r} \times N_{t}$ diagonal matrix with $\lambda_{k, 1} \geq \lambda_{k, 2} \geq \cdots \geq \lambda_{k, N_{B}}$, and $V_{k}$ is an $N_{t} \times M$ unitary matrix formed by the corresponding eigen-vectors $v_{k, i}$, i.e., $V_{k}=\left(v_{k, 1}, \cdots, v_{k, N_{B}}\right)$. The optimal unquantized precoder for each channel condition should be $\boldsymbol{F}_{\text {opt }}=\overline{\boldsymbol{V}}_{k}$, where $\overline{\boldsymbol{V}}_{k}$ is the matrix constructed from the first $M$ columns of $\boldsymbol{V}_{k}$ [11], [28]. Based on those decomposition matrices, we can derive the closed form solution for the power loading consequently, which is summarized in the following theorem. The proof is omitted in this paper.

Theorem 1: With optimal eigen-beamforming combined with power allocation, we get the optimal transmitter power allocation schemes by water-filling principle:

$$
\begin{aligned}
& \phi_{m}(k)=\frac{1}{\lambda_{m}(k)} \times \\
& {\left[\frac{P_{0}+\sum_{k=1}^{N_{B}} \sum_{m=1}^{\tilde{M}} \lambda_{m}(k)^{-1} \ln \lambda_{m}(k)}{\sum_{k=1}^{N_{B}} \sum_{m=1}^{\tilde{M}} \lambda_{m}^{-1}(k)}+2 \ln \lambda_{m}(k)\right]^{+}}
\end{aligned}
$$

where $(x)^{+}=\max (0, x), k \in\left[1, N_{B}\right]$, and $p_{m}(k)$ denotes the $m$ th entry of the diagonal power allocation matrix of the $k$ th region, and $\tilde{M}$ denote the number of active data streams in each subcarrier.

2) Solution of Step2: Determine the optimal region $\left\{R_{k}\right\}_{k=1}^{N_{B}}$, for a given transmission strategy $\left\{\rho_{k}\right\}_{k=1}^{N_{B}}$. The optimal partition region is given by the nearest neighbor rule. In our case, we will use the distortion measure as (26). Thus, we can get the updated regions by the following

$$
H_{k}=\left\{H: D\left(H, \rho_{k}\right) \leq D\left(H, \rho_{j}\right), \forall j \neq k\right\}
$$

The above two necessary conditions have been proved to be essential for the codebook construction [31]. Firstly, for each region, the optimal codeword can be chosen to minimize the distortion. In our case, multimode beamforming together with power allocation are employed in centriod condition. Secondly, the nearest neighbor rule is used to find the optimal region represented by each codeword.

3) Solution of Codewords Selection: Here, we present the optimal codeword selection algorithm for MIMO-OFDM system. By (4), our codeword selection algorithm can be employed on each sub-carrier. Let $\rho_{k}=\left\{w_{k}, \phi_{k}\right\}$ denotes the $k$ th codeword in codebook $\left\{\rho_{k}\right\}_{k=1}^{N_{B}}$. Then the description of the codeword selection is 
St1 : Obtain CSI by channel estimation at the receiver.

St2 : Perform the eigen decomposition (EVD): $\boldsymbol{w}^{H} \boldsymbol{H}^{H} \boldsymbol{H} \boldsymbol{w}=$ $\boldsymbol{W} \Lambda \boldsymbol{W}^{H}$, where $\Lambda$ is an $M \times M$ diagonal matrix with diagonal entries $\lambda_{k=1}^{N_{B}}$ and matrix $\boldsymbol{W}$ denote the eigen matrix of $\boldsymbol{w}^{H} \boldsymbol{H}^{H} \boldsymbol{H} \boldsymbol{w}$.

St3 : Let $D=\sum_{m=1}^{M} e^{-\dot{\phi}_{m} w^{H} H^{H} H w}$, where $M$ denotes the data stream over each subcarrier. The selected optimal codeword is given by

$$
\rho^{o p t}=\arg \min _{\rho \in W} D(H, \rho)
$$

where $W$ is the codebook.

\section{Simulation Result AND Discussion}

In this section, we provide some numerical examples to illustrate the performance of the optimal limited feedback design in section IV. We present Monte Carlo simulation for the MIMO-OFDM system with $N_{t}=4$ and $N_{r}=2$. The number of sub-carriers is 64 , the cyclic prefix length is 16 , and the used constellation is QPSK. We assumed that the discrete-time channel impulse response is generated according to the Hiperlan 2 Channel Model C in [30]. The channels between different transmit and receiver antennas are assumed independent. The channel is fixed for a frame and randomly varies between frames. $n(k)$ is an i.i.d complex Gaussian of zero mean. The transmitter power was allocated across subcarriers by water filling principle. The receiver uses linear decoder with perfect channel knowledge. It is assumed that the feedback CSI had no delay and no transmission error.

Experiment 1: The first experiment is about the convergence of the iterative descent algorithm. Fig. 2 shows a realization of the achieved distortion after each iteration. The codebook size is 64 . We can observe that the iterative algorithm converges very fast, typically after three to four times of iterations.

Experiment 2: The second experiment compares the BER performance of a 4 MIMO-OFDM system with ideal CSI and partial CSI at the transmitter. The number of data stream over each subcarrier is 2 . When the transmitter has perfect CSI, by using (7), the minimum BER based water-filling principle is employed. In partial CSI scenario, the transmitter can get partial CSI by limited feedback. We compare the performance of the existing codebook in [10]and [31] with our BERbased codebook. Fig. 3 shows that when the transmitter get perfect CSI, the system can get best BER performance; when the transmitter only get partial CSI, BER-based codebook can get moderate performance improvement over the existing codebooks. Fig. 3 also shows that the codebook in [10] is a little bit better than that in [31].

4) Experiment 3: The influence of size of the codebook for a $4 \times 2$ system with MMSE receiver is addressed in Fig. 4 . The cases $B=4,6,7,8$, corresponds to the codebook sizes $16,6,128,256$ respectively, and the perfect CSI is denoted by $B=\infty$. From Fig. 4 , we observe:

- Feedback link can improve the system performance dramatically;

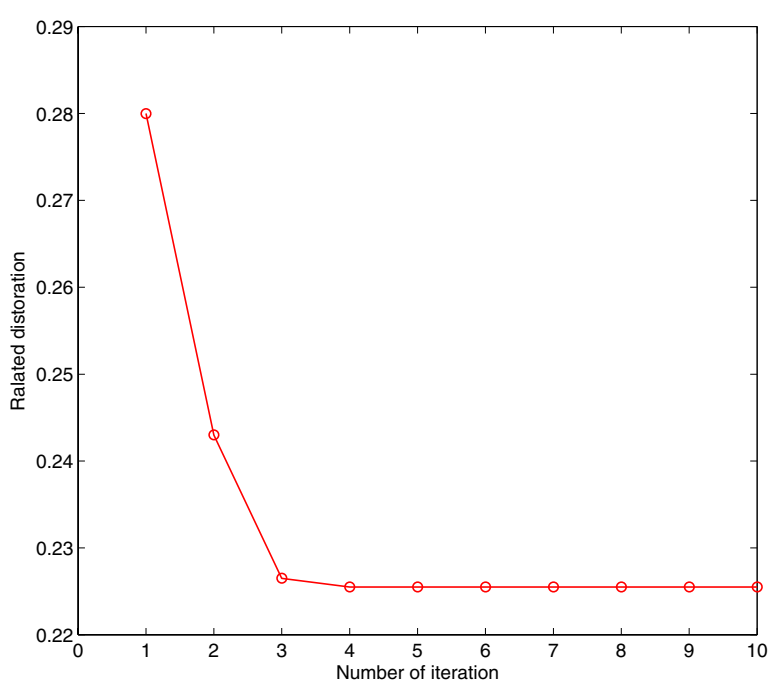

Fig. 2. Convergence of codebook construction algorithm

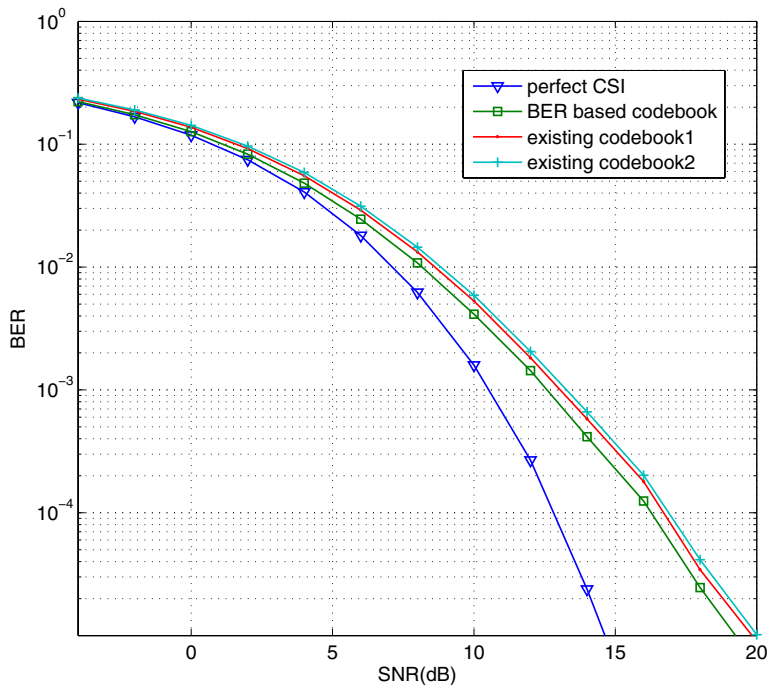

Fig. 3. BER performance of perfect CSI and limited CSI

- When the feedback bits increases, the system will have performance gain with $B=\inf$ as a performance benchmark.

- The gap between $B=6, B=7$ and $B=8$ is small, so the size of codebook in a practical system is not necessarily large.

\section{CONCLUSION}

In this paper, we consider the precoded spatial multiplexing MIMO-OFDM system with limited feedback of CSI. We propose a new BER based codebook construction algorithm, which outperforms the previous codebook of equal power allocation. We also develop the optimal algorithm for MIMOOFDM system with limited feedback of CSI. The essential 


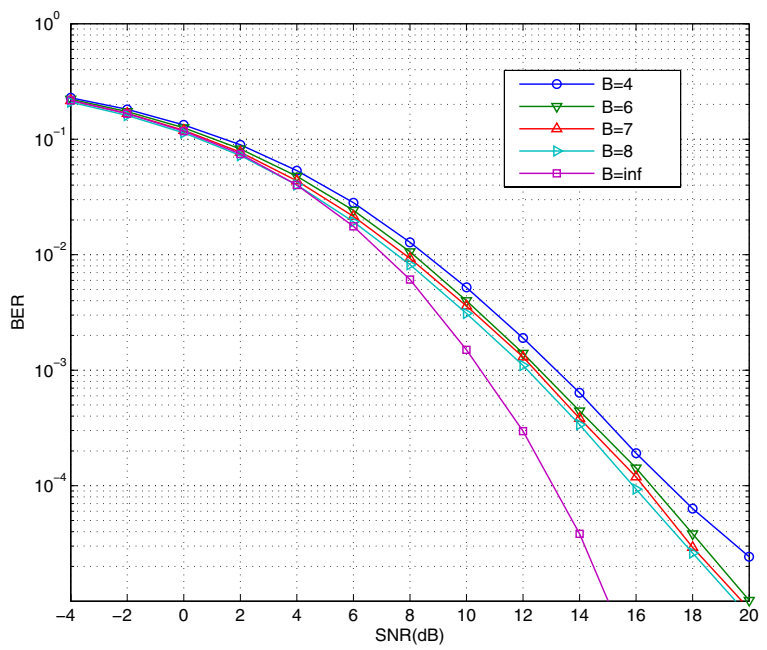

Fig. 4. The effect of codebook size

component of this paper is codebook design using optimal structure of the transmitter and receiver. In other words, we employ the optimal transmission structure which jointly combines multimode beamfoming and power allocation. Although the presented codebook design algorithm is complicated than that of previous works, we do not need to care about the complexity because the codebook is designed offline.

\section{ACKNOWLEDGMENT}

This work is supported by NSF China \#60572157 and \#60672067 by NSF Shanghai \#062R14041, by ShanghaiCanada NRC \#06SN07112, by Cultivation Fund of the Key Scientific and Technical Innovation Project, Ministry of Education of China \#706022, and by Program for New Century Excellent Talents in University \#NCET-06-0386.

The authors would like to thank Lei Chen and Xin Wang for their helpful discussion and some simulation works.

\section{REFERENCES}

[1] S. M. Alamouti, "A simple diversity techniques for wireless communications," IEEE J. Select. Areas commun., vol. 16, pp.1451-1458, Oct., 1998.

[2] V. Tarokh, N. Seshadri, and A. R. Calderbank, "Space-time codes for high data rate wireless communicatoin: performance analysis and code construction," IEEE Trans. Inform. Theory, vol. 44, pp. 744-765, Mar., 1998

[3] G. Yi and K. B. Letaidf, "Performance Evaluation and Analysis of Space-Time Coding in Unequalized Multipath Fading Links," IEEE trans. Commun., vol. 48, no. 11, Nov., 2000.

[4] V. Tarokh, N. Seshadri, and A.R. Calderbank, "A recursive algorithm for the exact BER computation of generalized hierarchical QAM constellations," IEEE Trans. Inform. Theory, vol. 49, no. 1, pp. 297-307, Jan., 2003.

[5] S. A. Jafar and A. Goldsmith, "Transmitter optimization and optimality of beamforming for multiple antenna systems," IEEE Trans. Wireless Commun., vol. 3, no. 4, Jul., 2004.

[6] P. Xia, G. B. Giannakis, "Design and Analysis of transmit-beamforming based on limited-rate feedback," IEEE Trans. signal. processing, vol. 54, no. 5, pp. 1853-1863, May., 2006.
[7] F. Rey, M.Lamarca, "Robust power allocation algorithms for MIMO OFDM system with imperfect CSI," IEEE Trans. signal. processing, vol. 53, no. 3, pp. 1070-1085, Mar., 2005.

[8] V. Mignone and A. Morello, "CD3-OFDM: A novel demodulation scheme for fix and mobile receivers," IEEE Trans. commun., vol. 44, no. 5, pp. 1144-1151, May., 1996.

[9] J. A. C. Bingham, "Multicarrier Modulation for Data Transmission: An idea whose time has come," IEEE Trans. Commun., vol.28, May, 1990.

[10] S. L. Zhou, "BER criterion and codebook construction for finite-rate precoded spatial multiplexing with linear receiver," IEEE Trans. signal processing, vol. 50, no. 10, pp. Oct., 2002.

[11] P. Xia, "Design and analysis of transmit-beamforming based on limitedrate feedback," IEEE Trans. Signal Processing. vol. 54, no. 5, pp. 18531863, May, 2006.

[12] D. J. Love and R. W. Heath Jr., "Grassmannian precoding for spatial multiplexing systems ," Allerton Conf. on Comm. Control and Comp., Monticello, IL, Oct., 2003.

[13] D. J. Love and R. W. Heath Jr., "Limited feedback precoding for spatial multiplexing systems," Proc. of Global Telecommunications Conference, San Francisco, CA, vol. 4, pp. 1857-1861, Dec., 2003.

[14] D. J. Love, R. W. Heath Jr., and T. Strohmer, "Grassmannian beamforming for multiple-input multiple-output wireless systems," IEEE Trans. Inform. Theory, vol. 49, no. 10, pp. 2735-2747, Oct., 2003.

[15] K. Cho and D. Yoon, "On the general BER expression of oneand twodimensional amplitude modulations, ”, IEEE Transactions on Communications, vol. 50, no. 7, pp. 1074-1080, July, 2002.

[16] A. Narula, M. J. Lopez, M. D. Trott, and G. W. Wornell, "Efficient use of side information in multiple-antenna data transmission over fading channels," IEEE J. Select. Areas Commun., vol. 16, no. 8, pp. 1423-1436, Oct., 1998.

[17] S. Zhou, Z. Wang, and G. B. Giannakis, "Quantifying the power-loss when transmit-beamforming relies on finite rate feedback," IEEE Trans. Wireless Commun., vol. 4, no. 4, pp. 1948-1957, Jul., 2005.

[18] M. Vu, A. Paulraj, "Optimal linear precoders for MIMO wireless correlated channels with nonzero mean in space-time coded systems," IEEE Trans. Signal Processing. vol. 54, No. 6, pp. 2318-2332, Jun., 2006.

[19] S. L. Zhou, "Optimal transmitter eigen-beamforming and space-time block coding based on channel mean feedback," IEEE trans. signal processing, vol. 50, no. 10, pp. 2599-2613, Oct., 2002

[20] D. P. Palomar, J. M. Cioffi, "Joint Tx-Rx beamforming design for multicarrier MIMO channels: a unified framework for convex optimization," IEEE Trans. signal processing, vol. 51, no. 9, pp. 2381-2401, Sep., 2003.

[21] A. Scaglione, P. Stoica, S. Barbarossa, "optimal design for space-time linear precoders and decoders," IEEE Trans. Signal Processing. Vol. 50, No. 5, pp. 1051-1064, May, 2002.

[22] H. Sampath, P. Stoica and A. Paulraj, "Generalized Linear Precoder and Decoder Design for MIMO Channels Using the Weighted MMSE Criterion," IEEE Trans. Commun., Vol. 49, no. 12, pp. 2198-2206, Dec., 2001.

[23] S. T. Chung and A. J. Goldsmith, "Degrees of freedom in adaptive modulation: A unified view," IEEE Trans. commun., vol. 49, no. 9, pp. 1561-1571, Mar., 2001.

[24] V. Lau, Y. Liu, and T.-A. Chen, "On the design of MIMO block-fading channels with feedback-link capacity constraint," IEEE Trans. Commun., vol. 52, no. 1, pp. 62-70, Jan., 2004.

[25] E. N. Onggosanusi, A. M. Sayeed, B. V. V. Veen, "Efficient signaling schemes for wideband space-time wireless channels using channels state information," IEEE Trans. Vehic Techn. Vol. 52, No. 1, pp. 1-13, May, 2003.

[26] N. J. A. Sloane. "Packings in Grassmannian spaces", [Online] Available: http://www.research.att.com/ njas/grass/index.html.

[27] A. Gersho and R. M. Gray, "Vector Quantization and Signal Compression," Kluwer Academic Publishers, Boston, 1992.

[28] D. J. Love, R. W. Heath Jr., "Limited feedback unitary for spatial multiplexing system," IEEE Trans. Inform Theory, vol. 51, no. 8, pp. 2967-2976, Aug., 2005.

[29] P. Xia, "Multi-input multi-output communications with partial channel state information," Ph.D. Dissertation, Dept. Elect. Eng., Minesota Univ., 2004.

[30] J. Medbo and P. Schramm, "Channel models for HIPERLAN/2 in different indoors scenarios," in ETSI/BRAN 3ERI085B, Mar., 1998.

[31] D. Love, Personal Webpage on Grassmannian Subspace Packing, Online: http://dynamo.ecn.purdue.edu/ djlove/grass.html. 